\title{
Gravoturbulent star formation: Effects of the equation of state on stellar masses
}

\author{
Ralf S. Klessen ${ }^{1}$, Marco Spaans ${ }^{2}$ and Anne-Katharina Jappsen ${ }^{1}$ \\ ${ }^{1}$ Astrophysikalisches Institut Potsdam, An der Sternwarte 16, 14482 Potsdam, Germany \\ email: rklessen@aip.de, akjappsen@aip.de \\ ${ }^{2}$ Kapteyn Astronomical Institute, P.O. Box 800, 9700 AV Groningen, The Netherlands \\ email: spaans@astro.rug.nl
}

\begin{abstract}
Stars form by gravoturbulent fragmentation of interstellar gas clouds. The supersonic turbulence ubiquitously observed in Galactic molecular gas generates strong density fluctuations with gravity taking over in the densest and most massive regions. Collapse sets in to build up stars and star clusters.

Turbulence plays a dual role. On global scales it provides support, while at the same time it can promote local collapse. Stellar birth is thus intimately linked to the dynamic behavior of parental gas clouds, which governs when and where protostellar cores form, and how they contract and grow in mass via accretion from the surrounding cloud material to build up stars. The equation of state plays a pivotal role in the fragmentation process. Under typical cloud conditions, massive stars form as part of dense clusters following the "normal" mass function observed, e.g. in the solar neighborhood. However, for gas with an effective polytropic index greater than unity star formation becomes biased towards isolated massive stars. This is relevant for understanding the properties of zero-metallicity stars (Population III) or stars that form under extreme environmental conditions like in the Galactic center or in luminous starbursts.
\end{abstract}

Keywords. stars: formation - methods: numerical - hydrodynamics - turbulence - equation of state - ISM: clouds - ISM: evolution

\section{Introduction}

Identifying the physical processes that determine the masses of stars and their statistical distribution, usually known as the initial mass function (IMF), is a fundamental problem in star formation research. Observations in the solar vicinity and in nearby young star clusters suggest the existence of a characteristic stellar mass $M_{\mathrm{ch}}$. The IMF peaks at this characteristic mass which is typically a few tenths of a solar mass. For larger masses the IMF has a nearly power-law form and it declines rapidly towards smaller masses (Scalo 1998; Kroupa 2002; Chabrier 2003).

Although the initial stellar mass function in our Milky Way has been derived from vastly different regions, ranging from the immediate neighborhood of our Sun to dense and distant stellar clusters, its key features seem to be strikingly universal to all determinations (e.g. Kroupa 2001, Meyer et al. 2004 and references therein). However, the environmental conditions in the considered regions vary enormously. If the formation of stars and the resulting mass spectrum strongly depend on initial conditions, there would thus be no reason for the IMF to be universal. A derivation of the characteristic stellar mass that is based on fundamental atomic and molecular physics would therefore be highly desirable.

On the other hand, there are hints for IMF variations in more extreme environments and/or at different metallicities. For example, HST/WFPC2 observations of the LMC 
by Gouliermis, Brander \& Henning (2004) indicate that local conditions seem to favor the formation of higher mass stars (top-heavy IMF) in associations, and not in the background field. Similar conclusions may be drawn for the stellar mass spectrum in the Galactic Center (Mark Morris, this proceedings; or Stolte et al. 2003 for the Arches Cluster) or in starburst galaxies (e.g. Elmegreen 2004, and references therein).

There are various ways to approach the formation of stars and star clusters from a theoretical point of view. Currently, the most successful models are based on the interplay between self-gravity and the interstellar turbulence ubiquiteously observed in Galactic molecular clouds. In this picture, stars form by a process we call gravoturbulent fragmentation (see, e.g. the reviews by Larson 2003, or Mac Low \& Klessen 2004). However, despite a wealth of observational data for the Milky Way (e.g. Fuller \& Myers 1992; Goodman et al. 1998), the nature of interstellar turbulence and the thermodynamic state of the star-forming gas (as characterized by the equation of state - EOS) remain major theoretical problems in understanding the stability and collapse of molecular clouds. The stiffness of the EOS can be largely responsible for the resulting density probability function of interstellar gas in the turbulent ISM. In particular, the value of the polytropic index $\gamma$ when adopting an EOS of the form $P \propto \rho^{\gamma}$ strongly influences the compressibility of density condensations as well as the amount of clump fragmentation. Hence, the EOS is directly related to the IMF (Vázquez-Semadeni et al. 1996; Li, Klessen, \& Mac Low 2003; Jappsen et al. 2005). In Spaans \& Silk $(2000,2005)$ the properties of a polytropic EOS were investigated and it was found that the stiffness of the EOS depends strongly on the ambient metallicity and the infrared background radiation field produced by irradiated dust grains. It thus may vary considerably in different galactic environments.

The structure of this paper is as follows: In the next section, $\S 2$, we briefly review the basic concepts of gravoturbulent fragmentation and discuss its relation to stellar birth. In $\S 3$ we consider the fragmentation behavior of self-gravitating, supersonically turbulent gas with a polytropic EOS. Small values of the polytropic exponent $\gamma$ lead to the formation of dense clusters of low-mass stars, while $\gamma>1$ introduces a bias towards more massive and isolated stars. In $\S 4$, we discuss the thermal properties of the star-forming gas in our Galaxy and their implications for the IMF in the solar neighborhood. Finally, in $\S 5$ we present first numerical calculations of gravoturbulent fragmentation in starburst galaxies suggesting that the IMF in very extreme environments may be top-heavy.

\section{The Concept of Gravoturbulent Fragmentation}

Supersonic turbulence plays a dual role in star formation. While it usually is strong enough to counterbalance gravity on global scales it will usually provoke collapse locally. This process is discussed in detail in the reviews by Larson (2003) and Mac Low \& Klessen (2004). Turbulence establishes a complex network of interacting shocks, where regions of high-density build up at the stagnation points of convergent flows. These gas clumps can be dense and massive enough to become gravitationally unstable and collapse when the local Jeans length becomes smaller than the size of the fluctuation. However, the fluctuations in turbulent velocity fields are highly transient. They can disperse again once the converging flow fades away (Vázquez-Semadeni et al. 2005). Even clumps that are strongly dominated by gravity may get disrupted by the passage of a new shock front (Mac Low et al. 1994).

For local collapse to result in the formation of stars, Jeans unstable, shock-generated, density fluctuations must collapse to sufficiently high densities on time scales shorter than the typical time interval between two successive shock passages. Only then do they 'decouple' from the ambient flow pattern and survive subsequent shock interactions. The 
shorter the time between shock passages, the less likely these fluctuations are to survive. The overall efficiency of star formation depends strongly on the wavelength and strength of the driving source (Klessen et al. 2000; Heitsch et al. 2001). Both regulate the amount of gas available for collapse on the sonic scale where turbulence turns from supersonic to subsonic (Vázquez-Semadeni et al. 2003).

The velocity field of long-wavelength turbulence is dominated by large-scale shocks which are very efficient in sweeping up molecular cloud material, thus creating massive coherent structures. These exceed the critical mass for gravitational collapse by far. The situation is similar to localized turbulent decay, and quickly a cluster of protostellar cores builds up (e.g. Klessen et al. 1998; Klessen 2001; Bate et al. 2003; Li et al. 2004; or Bonnell, this proceedings). A prominent example is the Trapezium Cluster in Orion with a few thousand young stars (Hillenbrand 1997). However, this scenario also applies to the Taurus star-forming region (Hartmann 2002) which is historically considered as a case of isolated stellar birth. Its stars have formed almost simultaneously within several coherent filaments which apparently are created by external compression (see Ballesteros-Paredes et al. 1999). This renders it a clustered star-forming region in the sense of the above definition.

The efficiency of turbulent fragmentation is reduced if the driving wavelength decreases. There is less mass at the sonic scale and the network of interacting shocks is very tightly knit. Protostellar cores form independently of each other at random locations throughout the cloud and at random times. There are no coherent structures with multiple Jeans masses. Individual shock generated clumps are of low mass and the time interval between two shock passages through the same point in space is small. Hence, collapsing cores are easily destroyed again. Altogether star formation is inefficient, and stars are dispersed throughout the cloud.

\section{Gravoturbulent Fragmentation in Polytropic Gas}

As indicated above, the thermodynamic properties of the star-forming gas may play a crucial role in determining the stellar mass spectrum. As a first and crude approximation, the balance between heating and cooling in a molecular cloud can be described by a polytropic EOS, $P=K \rho^{\gamma}$, where $K$ is a constant, and $P, \rho$ and $\gamma$ are thermal pressure, gas density, and polytropic exponent, respectively. Depending on the environment, the exponent $\gamma$ can cover the range $0.2<\gamma<1.4$ in the interstellar medium (e.g. Spaans \& Silk 2000, 2005; Scalo \& Biswas 2002).

Li et al. (2003) carried out detailed numerical calculations to determine the effects of different EOS's on gravoturbulent fragmentation by varying $\gamma$ in steps of 0.1 in otherwise identical simulations. Figure 1 illustrates how a low exponent $\gamma$ leads to the build-up of a dense cluster of stars, while high values of $\gamma$ result in isolated star formation. It also shows that the spectra of both the gas clumps and protostars change with $\gamma$. In low- $\gamma$ models, the mass distribution of the collapsed protostellar cores at the high-mass end is roughly log-normal. As $\gamma$ increases, fewer but more massive cores emerge. When $\gamma>1.0$, the distribution is dominated by high mass protostars only, and the spectrum tends to flatten out. It is no longer described by either a log-normal or a power-law. The clump mass spectra, on the other hand, do show power-law behavior at the high mass side, even for $\gamma>1.0$.

This suggests that in a low- $\gamma$ environment stars tend to form in clusters and with small masses. On the other hand, massive stars can form in small groups or in isolation in gas with $\gamma>1.0$. 

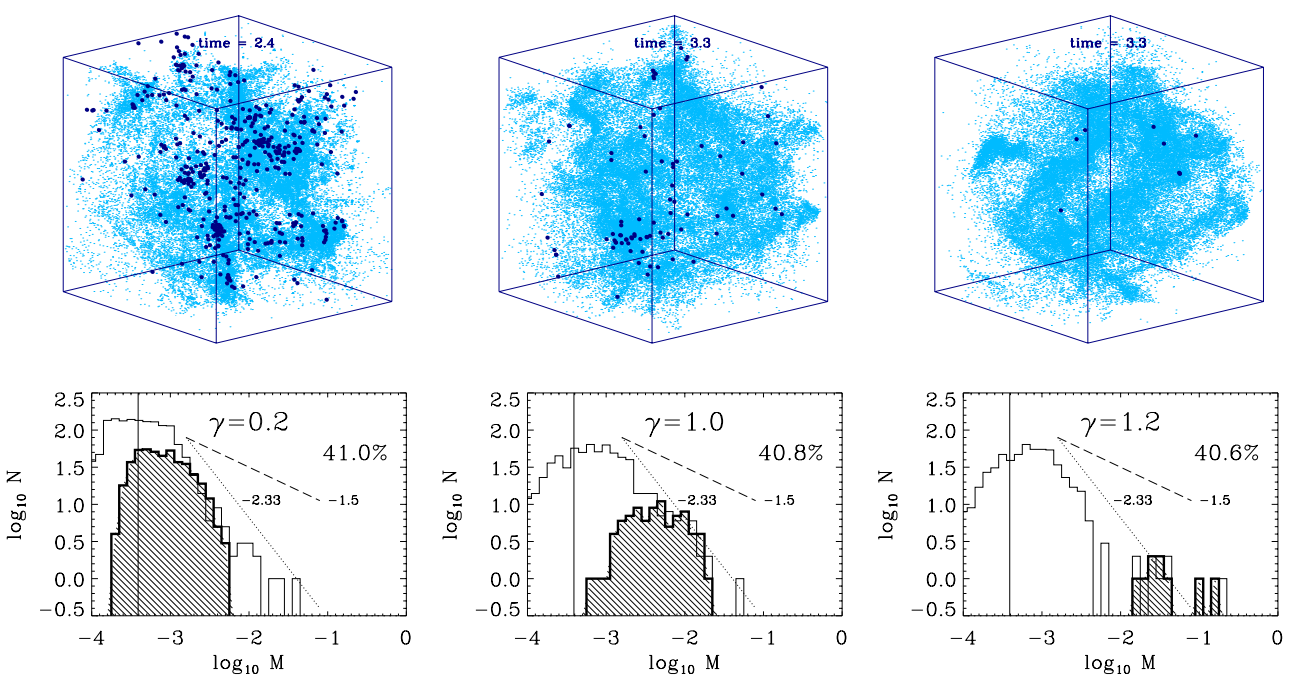

Figure 1. Top: 3-D distribution of the gas and protostars for different $\gamma$. Bottom: Mass spectra of gas clumps (thin lines) and of protostars (collapsed cores: hatched thick-lined histograms) for the corresponding cube above. The percentage shows the fraction of total mass accreted onto protostars. The vertical line shows the numerical resolution limit. Shown also are two power-law spectra with $\nu=-1.5$ (dashed-line) and $\nu=-2.33$ (dotted line). Figure adopted from Li et al. (2003).

The usefulness of theoretical predictions for the formation of isolated massive stars may be debated, since usually, massive stars are found in clusters. It is therefore interesting to note, that recently Lamers et al. (2002) reported observations of isolated massive stars or very small groups of massive stars in the bulge of M51. Also Massey (2002) finds massive, apparently isolated field stars in both the Large and Small Magellanic Clouds. This is consistent with our models assuming $\gamma>1.0$.

\section{Thermal Properties of Galactic Gas and Implications for the IMF}

Observational evidence indicates that dense prestellar cloud cores show a rough balance between gravity and thermal pressure (Benson \& Myers 1989; Myers et al. 1991). Thus, the thermodynamic properties of the gas play an important role in determining how dense star-forming regions in molecular clouds collapse and fragment. Observational and theoretical studies of the thermal properties of collapsing clouds both indicate that at densities below $10^{-18} \mathrm{~g} \mathrm{~cm}^{-3}$, roughly corresponding to a number density of $n=2.5 \times$ $10^{5} \mathrm{~cm}^{-3}$, the temperature decreases with increasing density. This is due to the strong dependence of molecular cooling rates on density (Koyama \& Inutsuka 2000). Therefore, the polytropic exponent $\gamma$ is below unity in this density regime. At densities above $10^{-18} \mathrm{~g} \mathrm{~cm}^{-3}$, the gas becomes thermally coupled to the dust grains, which then control the temperature by far-infrared thermal emission. The balance between compressional heating and thermal cooling by dust causes the temperature to increase again slowly with increasing density. Thus the temperature-density relation can be approximated with $\gamma$ above unity in this regime (Larson 1985, 2005; Spaans \& Silk 2000, 2005). Changing $\gamma$ from a value below unity to a value above unity results in a minimum temperature at the critical density. As shown by Li et al. (2003), gas fragments efficiently for $\gamma<1.0$ and less efficiently for higher $\gamma$. Thus, the Jeans mass at the critical density defines a characteristic mass for fragmentation, which may be related to the peak of the IMF. 


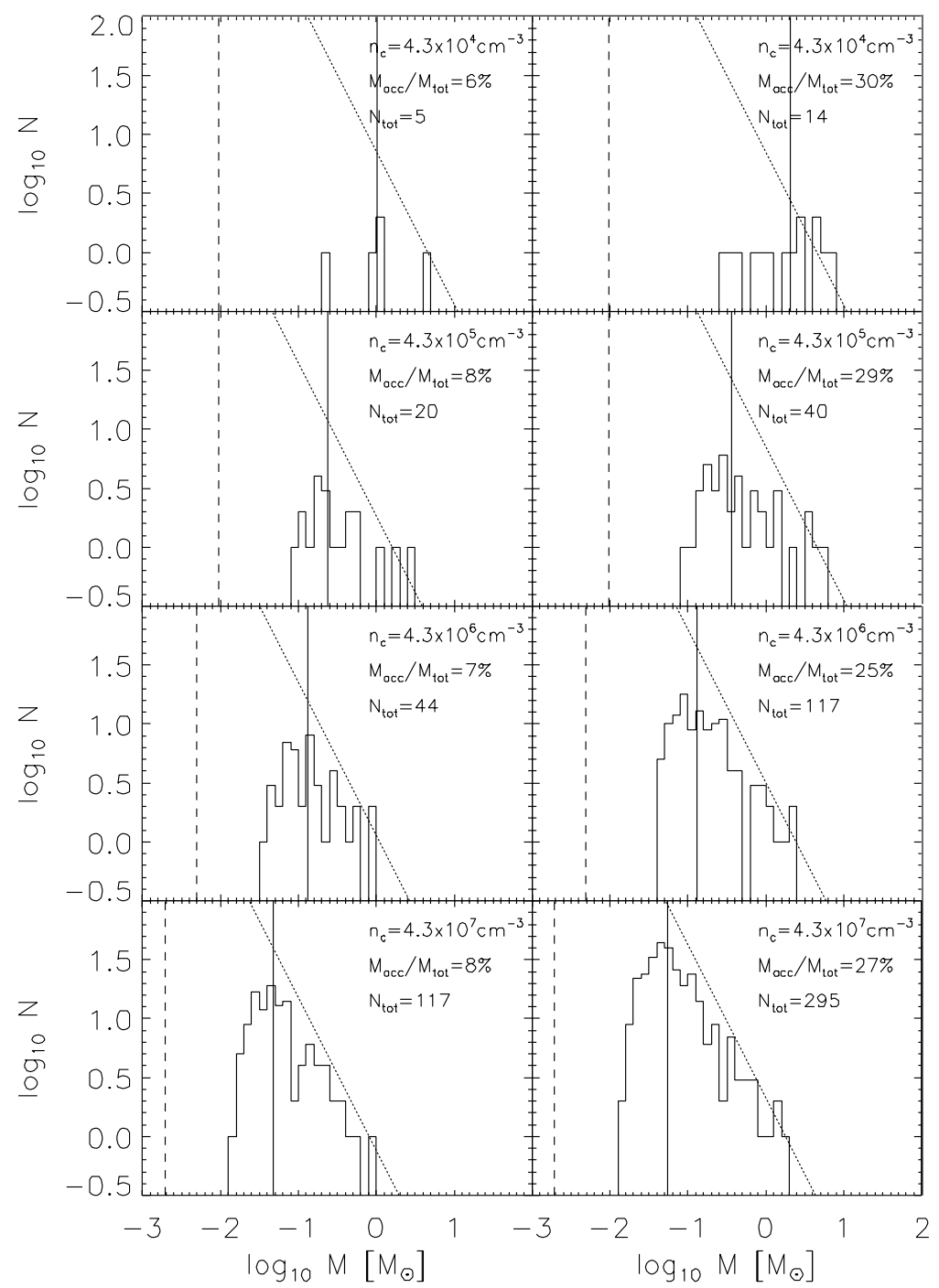

Figure 2. Mass spectra of protostellar cores for four models with critical densities in the range $4.3 \times 10^{4} \mathrm{~cm}^{-3} \leqslant n_{\mathrm{c}} \leqslant 4.3 \times 10^{7} \mathrm{~cm}^{-3}$. We show two phases of evolution, when about $10 \%$ and $30 \%$ of the total mass has been accreted onto protostars. The vertical solid line shows the position of the median mass. The dotted line serves as a reference to the Salpeter value (Salpeter 1955). The dashed line indicates the mass resolution limit. (Adopted from Jappsen et al. 2005.)

This hypothesis has been tested by Jappsen et al. (2005), who studied the effects of using a piecewise polytropic EOS with a polytropic exponent that changes from $\gamma=0.7$ to $\gamma=1.1$ at critical densities in the range $4.3 \times 10^{4} \mathrm{~cm}^{-3} \leqslant n_{\mathrm{c}} \leqslant 4.3 \times 10^{7} \mathrm{~cm}^{-3}$. Figure 2 illustrates how the characteristic mass $M_{\text {ch }}$ for fragmentation depends on the adopted critical density. Closest correspondence with the observed IMF (see, Scalo 1998; Kroupa 2002; Chabrier 2003) occurs for a critical density of $4.3 \times 10^{6} \mathrm{~cm}^{-3}$ and for stages of accretion around 30\% and above. At high masses, the distribution of collapsed objects follows a Salpeter-like power law. For comparison we indicate the Salpeter slope $x \approx 1.3$ (Salpeter 1955) where the IMF is defined by $\mathrm{d} N / \mathrm{d} \log m \propto m^{-x}$. For masses around the median mass the distribution exhibits a small plateau and then falls off towards smaller 
masses. The top-row model where the change in $\gamma$ occurs below the initial mean density, shows a flat distribution with only a few, but massive cores. They reach masses up to $10 \mathrm{M}_{\odot}$ and the minimal mass is about $0.3 \mathrm{M}_{\odot}$. The distribution becomes more peaked for higher $n_{\mathrm{c}}$ and there is a shift to lower masses.

Altogether, the characteristic mass $M_{\text {ch }}$ decreases with increasing critical density $n_{\mathrm{c}}$ following the relation $M_{\mathrm{ch}} \propto n_{\mathrm{c}}^{-0.5 \pm 0.1}$. The density at which $\gamma$ changes from below unity to above unity thus selects a characteristic mass scale for fragmentation. For physical parameters appropriate for the local interstellar medium, the resulting mass spectrum exhibits striking similarities to the observed IMF.

\section{The IMF in Starburst Galaxies}

In this section, we present a preliminary and first approach to understanding the IMF in starburst galaxies using direct numerical simulations of gravoturbulent cloud fragmentation.

Again, we employ a polytropic EOS, $P \propto \rho^{\gamma}$, where $\gamma$ is the polytropic index and $\rho$ the mass density. We adopt a perfect gas equation of state, $P \propto \rho T_{g}$ for the gas temperature $T_{g}$, to write $\gamma$ as

$$
\gamma=1+\frac{d \log T_{g}}{d \log \rho}
$$

This last step is justified (Scalo \& Biswas 2002; Vázquez-Semadeni, Passot \& Pouquet, 1996) as long as the heating and cooling terms in the fluid energy equation can adjust to balance each other on a time-scale shorter than the time-scale of the gas dynamics (i.e., thermal equilibrium). The model described in Spaans \& Silk $(2000,2005)$ is used in this work and the interested reader is referred to these papers for a detailed description of the various heating and cooling terms that influence the polytropic index.

The EOS was computed for self-gravitating spherical clouds approximated as singular isothermal spheres (Neufeld, Lepp \& Melnick 1995), for which the total hydrogen number density $n_{\mathrm{H}}$ scales with column density $N_{\mathrm{H}}$ per unit of velocity as

$$
N_{\mathrm{H}}=7.2 \times 10^{19} n_{\mathrm{H}}^{0.5} \mathrm{~cm}^{-2} /\left(\mathrm{km} \mathrm{s}^{-1}\right) .
$$

For the starburst environments of interest here, we assume a cosmic ray ionization rate of $3 \times 10^{-15} \mathrm{~s}^{-1}$. Solar relative abundances are assumed (Asplund et al., 2004; Jenkins 2004) while the overall metallicity is two times solar (Barthel 2004). We further adopt a MRN grain size distribution (Mathis, Rumpl \& Nordsieck 1977) and assume that the dust abundance scales with the carbon abundance. For the starburst model $\Delta V_{\text {tur }}=3$ $\mathrm{km} / \mathrm{s}$ is adopted to take the larger input of kinetic energy (e.g. through supernovae) into account. The dust temperature inside the model clouds is set by a fiducial background star formation rate of $100 M_{\odot} \mathrm{yr}^{-1}$ which causes dust to be at temperatures of about $T_{d}=70$ to $T_{d}=30 \mathrm{~K}$, depending on the amount of shielding. These values are consistent with dust temperatures determined by continuum observations of luminous infrared galaxies (Spinoglio et al., 2002; Klaas et al. 2001, 1997). No freeze-out of molecules is assumed. The latter assumption is justified because our dust is generally warmer than $20 \mathrm{~K}$.

An overabundance, relative to solar, of oxygen leads to a significantly stiffer EOS because the opacity in oxygen bearing molecules like CO and water is higher. This increases the amount of line trapping in these opaque cooling lines. Furthermore, when the temperature of the dust is high (larger than $30 \mathrm{~K}$ ), $\mathrm{H}_{2} \mathrm{O}$ collisional de-excitation heating dominates the thermal budget because the water levels are pumped by far-infrared dust emission. This causes the gas heating to attain a $n_{\mathrm{H}}^{2}$ density dependence (Takahashi, 


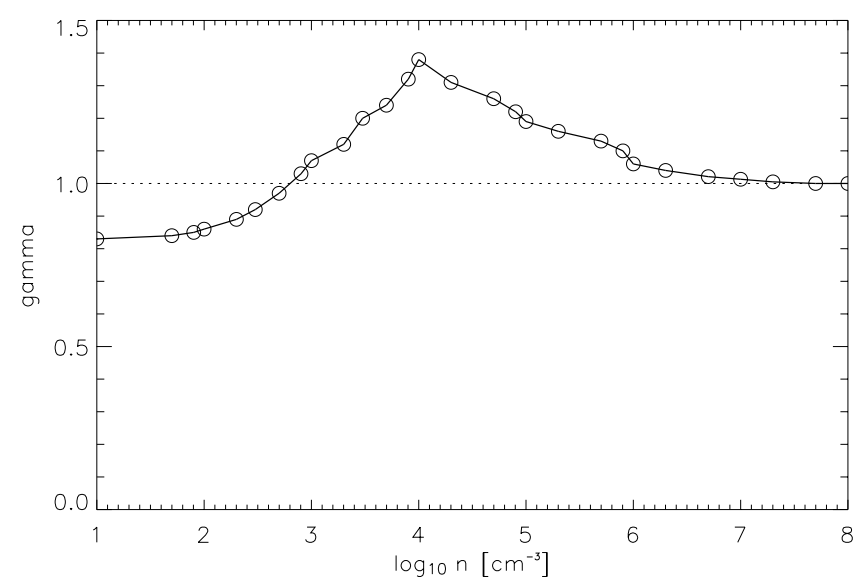

Figure 3. Starburst EOS adopted from Spaans \& Silk (2005).

Hollenbach \& Silk 1983; Spaans \& Silk 2000, 2005). Consequently, one has a strong peak, $\gamma \approx 1.4$, around a density of $\sim 10^{4} \mathrm{~cm}^{-3}$. Since the dust grains are warm, gas-dust heating around $10^{5} \mathrm{~cm}^{-3}$ causes the EOS to remain above unity, $\gamma \approx 1.1$, beyond the $\mathrm{H}_{2} \mathrm{O}$ opacity peak. Note here that gas-dust heating/cooling scales as $\left(T_{g}-T_{d}\right) T_{g}^{0.5} n_{\mathrm{H}}^{2}$. Hence, a starburst has a stiff EOS because of its high absolute metallicity and warm, $T_{d} \sim 30-70$ K, dust. Figure 3 shows the resulting EOS and the fit that has been adopted for the hydrodynamic simulation.

We adopt this EOS to model gravoturbulent star formation in a circumnuclear starburst environment using smoothed particle hydrodynamics ( $\mathrm{SPH})$. This is a Lagrangian method to solve the equations of hydrodynamics, where the fluid is represented by an ensemble of particles, and flow quantities are obtained by averaging over an appropriate subset of SPH particles (Benz 1990; Monaghan 1992). We use the parallel code GADGET, designed by Springel et al. (2001). The method is able to resolve large density contrasts as particles are free to move, and so the particle concentration increases naturally in highdensity regions. To determine the resolution limit of our calculation we deploy a criterium proposed by Bate \& Burkert (1997). It requires the local Jeans mass at all densities to be represented by at least 100 particles, and is adequate for the problem considered here, where we follow the evolution of highly nonlinear density fluctuations created by supersonic turbulence. Our version of the code furthermore replaces high-density cores with sink particles (Bate, Bonnell, \& Price 1995). Sink particles can accrete gas from their surroundings while keeping track of mass and momentum. This enables us to follow the dynamic evolution of the system over many local free-fall timescales.

We focus on a cubic molecular cloud region of $11.2 \mathrm{pc}$ in size. The region contains $80000 \mathrm{M}_{\odot}$ of gas and has a mean particle density $n=1000 \mathrm{~cm}^{-3}$. We drive turbulence uniformly on large scales, with wave numbers $k$ in the range $1 \leqslant k \leqslant 2$ as described in Mac Low (1999). The energy input rate is adjusted to yield a constant turbulent Mach number $\mathcal{M}_{\mathrm{rms}} \approx 5$. The particle number in the simulation presented here is $N=$ 8000000 , which is thus one of the highest resolution star-formation calculation ever done with SPH. The critical density for sink particle formation is $n_{c}=10^{7} \mathrm{~cm}^{-3}$. The mass of individual $\mathrm{SPH}$ particles is $m=0.01 \mathrm{M}_{\odot}$ which is sufficient to resolve the minimum Jeans mass in the system $M_{\mathrm{J}} \approx 1.5 \mathrm{M}_{\odot}$. Except for the EOS and the particle number, the numerical set-up is identical to the study by Jappsen et al. (2005). 


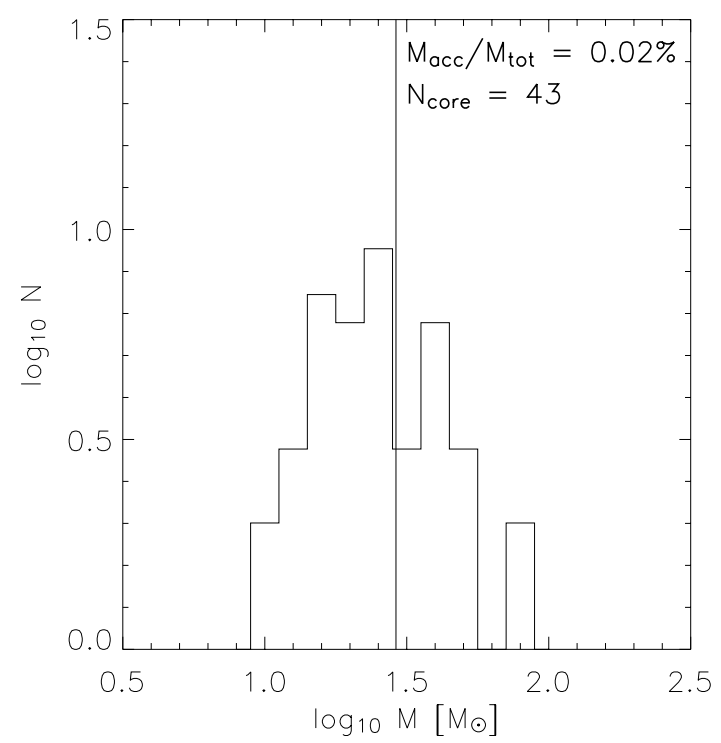

Figure 4. Mass spectrum of protostellar objects in a simulation focusing on a starburst environment. The size of the considered molecular cloud region is $11.2 \mathrm{pc}$ and it contains $80000 \mathrm{M}_{\odot}$. The system is depicted at early times when about $0.02 \%$ of the available gas is converted into stars. Comparison with Fig. 2 shows that the average mass (indicated by the vertical line) is shifted towards high values. The nominal resolution limit lies at $1 \mathrm{M}_{\odot}$.

As a preliminary result, we find that in the considered starburst galaxy, the mass spectrum of protostellar objects is biased towards high masses. This is illustrated in Fig. 4. The calculation thus supports the hypothesis that for extreme environmental conditions such as inferred for very dusty and IR-luminous circumnuclear starbursts the stellar initial mass function may indeed be top-heavy.

\section{Acknowledgements}

We thank Javier Ballesteros-Paredes, Peter Bodenheimer, Spyros Kitsionas, Richard Larson, Yuexing Li, Mordecai Mac Low, Stefan Schmeja, Joe Silk, and Enrique VázquezSemadeni for stimulating discussions and collaborations. RSK acknowledges support by the Emmy Noether Program of the Deutsche Forschungsgemeinschaft (grant no. KL1358/1).

\section{References}

Asplund, M., Grevesse, N., \& Sauval, J., 2004, astro-ph/0410214

Ballesteros-Paredes, J., Hartmann, L., \& Vázquez-Semadeni, E., 1999, ApJ, 527, 285

Barthel, P.D., 2005, in "The Dusty and Molecular Universe: A Prelude to Herschel and ALMA", ed. A. Wilson, (ESA SP-577, Noordwijk, Netherlands: ESA Publications Division), 41

Bate, M.R. \& Burkert, A. 1997, MNRAS, 288, 1060

Bate, M.R., Bonnell, I.A., \& Price, N.M. 1995, MNRAS, 277, 362

Bate, M.R., Bonnell, I.A., \& Bromm, V. 2003, MNRAS, 339, 577

Benson, P.J., \& Myers, P.C. 1989, ApJS, 71, 89

Benz, W. 1990, in Numerical Modelling of Nonlinear Stellar Pulsations Problems and Prospects, ed. J.R. Buchler (Dordrecht: Kluwer), 269 
Brandl, B.R., \& Andersen, M. 2004, in "IMF at 50: The Initial Mass Function 50 Years Later", eds. E. Corbelli, F. Palla, \& H. Zinnecker (Kluwer Academic Publisher), p. 237

Chabrier, G. 2003, PASP, 115, 763

Elmegreen, B.G. 2005, in "ASSL Vol. 329: Starbursts: from 30 Doradus to Lyman Break Galaxies", eds. R. de Grijs \& R. M. Gonzales Delgado (Kluwer), 57

Fuller, G.A. \& Myers, P.C., 1992, ApJ, 384, 523

Goodman, A.A., Barranco, J.A., Wilner, D.J., \& Heyer, M.H., 1998, ApJ, 504, 223

Gouliermis, D., Brander, W., \& Henning, Th., 2004, ApJ, 623, 846

Hartmann, L. 2002, ApJ, 578, 914

Hillenbrand, L.A.: 1997. AJ, 113, 1733

Jappsen, A.-K., Klessen, R.S., Larson, R.B., Y., L., \& Mac Low, M.-M. 2004, A $\& A, ~ 435,611$

Jenkins, E.B., in 'Origin and Evolution of the Elements', p. 339

Klaas, U., Haas, M., Heinrichsen, I., \& Schulz, B., 1997, A\&A, 325, L21

Klessen, R.S. 2001, ApJ, 556, 837

Klessen, R.S., Burkert, A., \& Bate, M.R. 1998, ApJ, 501, L205

Klessen, R.S., Heitsch, F., \& Mac Low, M.-M. 2000, ApJ, 535, 887

Koyama, H. \& Inutsuka, S. 2000, ApJ, 532, 980

Kroupa, P. 2001, MNRAS, 322, 231

Kroupa, P. 2002, Science, 295, 82

Lada, C.J., \& Lada, E.A. 2003, ARAA, 41, 57

Lamers, H.J.G.L.M., et al., 2002, ApJ, 566, 818

Larson, R.B. 1981, MNRAS, 194, 809

Larson, R.B. 1985, MNRAS, 214, 379

Larson, R.B. 2003, Rep. Prog. Phys., 66, 1651

Larson, R.B. 2005, MNRAS, 359, 211

Li, Y., Klessen, R.S., \& Mac Low, M.-M. 2003, ApJ, 592, 975

Li, P. S., Norman, M. L., Mac Low, M.-M., \& Heitsch, F. 2004, ApJ, 605, 800

Mac Low, M.-M. 2004, ApJ, 524, 169

Mac Low, M.-M. \& Klessen, R.S. 2004, Rev. Mod. Phys., 76, 125

Mac Low, M.-M., McKee, C. F., Klein, R. I., Stone, J.M., \& Norman, M.L., 1994, ApJ, 433, 757

Massey, P., ApJS, 141, 81

Mathis, J.S., Rumpl, W., \& Nordsieck, K.H., 1977, ApJ, 217, 425

Meyer, M.R., Greissl, J., Kenworthy, M., \& McCarthy, D., 2004, in "IMF at 50: The Initial Mass Function 50 Years Later", eds. E. Corbelli, F. Palla, \& H. Zinnecker (Kluwer), p. 245

Monaghan, J.J. 1992, ARAA, 30, 543

Myers, P.C., Fuller, G.A., Goodman, A.A., \& Benson, P.J. 1991, ApJ, 376, 561

Neufeld, D.A., Lepp, S., \& Melnick, G.J., 1995, ApJS, 100, 132

Padoan, P. 1995, MNRAS, 277, 377

Padoan, P., \& Nordlund, A. 2002, ApJ, 576, 870

Padoan, P., Nordlund, A., \& Jones, B.J.T. 1997, MNRAS, 288, 145

Salpeter, E.E. 1955, ApJ, 121, 161

Scalo, J. 1998, in ASP Conf. Ser. 142: The Stellar Initial Mass Function, ed. G. Gilmore \& D. Howell (San Francisco: Astron. Soc. Pac.), 201

Scalo, J., \& Biswas, A., 2002, MNRAS, 332, 769

Spaans, M., \& Silk, J. 2000, ApJ, 538, 115

Spaans, M., \& Silk, 2005, ApJ, 626, 644

Spinoglio, L., Andreani, P., \& Malkan, M.A., 2002, ApJ, 572, 105

Springel, V., Yoshida, N., \& White, S.D.M. 2001, New Astronomy, 6, 79

Stolte, A., Grebel, E.K., Brandner, W., \& Figer, D.F. 2003, ASP Conf. Ser. 287: Galactic Star Formation Across the Stellar Mass Spectrum, 287, 433

Takahashi, T., Hollenbach, D.J., \& Silk, J., 1983, ApJ, 275, 145

Vázquez-Semadeni, E., Ballesteros-Paredes, J., \& Klessen, R.S., 2003, ApJ, 585, L131

Vázquez-Semadeni, E., Passot, T., \& Pouquet, A., 1996, ApJ, 473, 881

Vázquez-Semadeni, E., Kim, J., Shadmehri, M., \& Ballesteros-Paredes, J. 2005, ApJ, 618, 344 